\title{
A Novel PPAR $\gamma$ Agonist, SP1818, Shows Different Coactivator Profile with Rosiglitazone
}

\author{
Yun Sun Park, Jiwon ChOI, Kun-yong KIm, Jong-Seok Lim, Sukjoon Yoon*, and Young YANG* \\ Department of Life Science, Research Center for Women's Diseases, \\ Sookmyung Women's University, Seoul 140-742, Republic of Korea
}

(Received September 4, 2009; Revised September 17, 2009; Accepted September 18, 2009)

\begin{abstract}
Peroxisome proliferator-activated receptor $\gamma($ PPAR $\gamma$ ) is a ligand-activated transcription factor that is used as a target for anti-diabetic drug development. In a search for novel PPAR $\gamma$ agonists, the $\beta$-carboxyethyl-rhodanine derivative SP1818 was identified. We report here the characteristics of SP1818 as a selective PPAR $\gamma$ agonist. In transactivation assays, SP1818 selectively activated PPAR $\gamma$, but the degree of PPAR $\gamma$ stimulation was less than with $1 \mu \mathrm{M}$ rosiglitazone. SP1818 also stimulated glucose uptake in a concentration-dependent manner. The adipocyte differentiation markers adiponectin, scavenger receptor CD36 and aP2 were weakly induced by treatment with SP1818, and TRAP220 subunit was specifically recruited into PPAR $\gamma$ activated by rosiglitazone but not PPAR $\gamma$ activated by SP1818.
\end{abstract}

Keywords: Adipogenesis, Diabetes, Rosiglitazone

\section{INTRODUCTION}

The peroxisome proliferator-activated receptors (PPARs) belong to the nuclear receptor superfamily of ligand-activated transcription factors (Lehmann et al., 1997; Hauser et al., 2000). The PPAR $\gamma$ gene is transcribed from alternative promoters that give rise to two major protein isoforms, PPAR 1 and PPAR 2 (Zhu et al., 1995). Both isoforms are expressed most abundantly in adipocytes, and PPAR $\gamma 2$ is almost entirely adipocyte-specific (Vidal-Puig et al., 1996). PPAR $\gamma$ is structurally composed of a central DNA-binding domain, an amino-terminal activation domain, and a carboxyl-terminal ligand-binding domain (LBD) that is required for a ligand-dependent transactivation (AF-2) function (Zoete et al., 2007).

Thiazolidinediones such as pioglitazone and rosiglitazone are high-affinity ligands and agonists for PPAR $\gamma$ that are currently used in the treatment of type 2 diabetes mellitus (Chiarelli and Di Marzio, 2008). However, the use of thiazolidinediones has been associated with adverse effects including weight gain, increased adipogenesis, renal fluid retention, plasma volume expansion and, more re-

${ }^{*}$ Corresponding authors

Tel: +82-2-710-9415 (SJ Yoon), +82-2-710-9590 (Y Yang)

Fax: +82-2-2077-7322 (SJ Yoon), +82-2-2077-7322 (Y Yang)

E-mail: yoosj@sm.ac.kr (SJ Yoon), yyang@sm.ac.kr (Y Yang) cently, possible increased incidence of cardiovascular events (Berger et al., 2005; Nissen and Wolski, 2007). Because of the adverse effects of glitazones, the characterization of new PPAR $\gamma$ ligands that retain metabolic efficacy without exerting adverse actions is essential for the development of new therapeutic strategies for type 2 diabetes mellitus. Thus, in a screen for novel PPAR $\gamma$ agonists, we found SP1818.

\section{MATERIALS AND METHODS}

\section{Cell lines and reagents}

3T3-L1 cells and 293T cells obtained from the American Type Culture Collection (ATCC) were maintained in Dulbecco's Modified Eagle Medium (DMEM) containing 10\% fetal bovine serum (FBS). Deoxy- $\left[{ }^{3} \mathrm{H}\right]$-glucose was obtained from R\&D (Minneapolis, MN, USA) and a scintillation cocktail was obtained from PerkinElmer (Waltham, MA, USA). D-glucose was obtained from DUCHEFA Biochemie (St. Louis, MO, USA). Trizol was obtained from Takara Korea (Seoul, Korea). A reverse transcription system was obtained from Fermentas (Glen Burnie, MD, USA).

\footnotetext{
Transactivation assay

The pFA-Gal4-PPAR $\alpha$-LBD, pFA-PPAR $\delta$-LBD and pFA-
} 
PPAR $\gamma$-LBD expression plasmids were provided by Dr. Jung Hyeong Lee (Kangwon National University, Chuncheon, Korea). At $75-90 \%$ confluence, 293T cells were transiently co-transfected with one of the expression vectors for pFA-Gal4-PPAR-LBDs together with pFR-Luc using the calcium phosphate transfection method. Following a $24 \mathrm{~h}$ incubation, the cells were treated with various concentrations of SP1818 or rosiglitazone and incubated for $16 \mathrm{~h}$. Luciferase assays were performed using a dual-luciferase reporter assay system according to the instructions of the manufacturer (Promega, CA, USA), and the activity was determined in a Victor3 luminometer (PerkinElmer, Inc., MA, USA).

\section{Adipocyte differentiation and glucose uptake assay}

Glucose uptake was carried out as previously described with some modifications (Elsegood et al., 2009). Differentiation of the 3T3-L1 cells was induced by the addition of DMEM medium supplemented with $0.5 \mathrm{M}$ isobutylmethyIxanthine (SIGMA-ALDRICH KOREA, Seoul, Korea), $2 \mu \mathrm{M}$ dexamethasone (SIGMA-ALDRICH KOREA, Seoul, Korea), $1.7 \mu \mathrm{M}$ insulin (SIGMA-ALDRICH KOREA, Seoul, Korea), and $10 \%$ FBS. The induction medium was removed after two days. Thereafter, the medium was changed every two days with DMEM containing $10 \%$ FBS. At the time of the experiments, $90 \%$ of the 3T3-L1 cells were filled with multiple lipid droplets. Fully differentiated cells were incubated for $12 \mathrm{~h}$ with various concentrations of the compounds. For the glucose transport assay, cells were washed three times with $1 \mathrm{ml}$ of prewarmed transport buffer $(140 \mathrm{mM}$ $\mathrm{NaCl}, 5 \mathrm{mM} \mathrm{KCl}, 1 \mathrm{mM} \mathrm{CaCl}$, $2.5 \mathrm{mM} \mathrm{MgCl}_{2}, 20 \mathrm{mM}$ TrisHEPES pH 7.4) followed by incubation in the presence or absence of various concentration of either SP1818 or rosiglitazone. After 30 min of incubation, cells were treated with a mixture of $5 \mathrm{mM}$ D-glucose and $2 \mu \mathrm{Ci} 2-\left[{ }^{14} \mathrm{C}(\mathrm{U})\right] \mathrm{de}-$ oxy-D-glucose followed by incubation for $10 \mathrm{~min}$ at $37^{\circ} \mathrm{C}$. The reaction was stopped by the addition of $0.25 \mathrm{ml}$ of 50 $\mathrm{mM} \mathrm{NaOH}$. The cell lysates were collected in a glass scintillation vial containing $3 \mathrm{ml}$ of a scintillation cocktail. After proper vortexing of the cell lysates and the scintillation cocktail, each vial was subjected to ${ }^{14} \mathrm{C}$ scintillation counting. Glucose uptake values were normalized against total protein values measured from lysates extracted in $\mathrm{NaOH}$. The values were also corrected for non-carrier mediated transport by measuring glucose uptake in the presence of $0.01 \mathrm{mM}$ of cytochalasin B.

\section{Reverse transcriptase-polymerase chain reaction}

Total RNA from differentiated 3T3-L1 cells was prepared using Trizol (Takara Korea, Seoul, Korea) and $3 \mu \mathrm{g}$ of total RNA was reverse transcribed using M-MuLV reverse transcriptase (Fermentas, MD, USA) at $37^{\circ} \mathrm{C}$ for $1 \mathrm{~h}$. PCR for the amplification of aP2, CD36, and adiponectin was then performed using a primer pair (mouse aP2: forward 5'-GCCAGGGACTTTGGGTACGTGTG-3', reverse 5'GGCCTTGAGGGCCTCGGTGAGA-3; mouse CD36 forward 5'-ATGGGCTGTGATCGGAACTG-3', reverse 5'-TG TGGTGCAGCTGCTACAGC-3; mouse adiponectin: forward 5'-CGGAATTCGGCATCCCAGGACA', reverse 5'-CACAT AAGCGGCTTCAAGCTTCG-3). PCR was performed with amplification cycles of $30 \mathrm{~s}$ of denaturation at $94^{\circ} \mathrm{C}, 30 \mathrm{~s}$ of annealing at $55^{\circ} \mathrm{C}$, and $30 \mathrm{~s}$ of extension at $72^{\circ} \mathrm{C}$. $\beta$-actin was used as the control.

\section{Mammalian two-hybrid assay}

A mammalian two-hybrid assay was performed as described previously with some modifications Expression vectors for pVP-PPAR $\gamma$ (DEF), pM-SRC-1, pM-TIF2, pMAIB-1, pM-p300, pM-TRAP220 and a reporter plasmid pFR-LUC were kindly provided by Dr. Shigeaki Kato (University of Tokyo, Tokyo, Japan). HEK 293T cells were transiently co-transfected with one of the expression vectors for pM-SRC-1, pM-TIF2, pM-AIB-1, pM-p300 or pMTRAP220 together with pVP-PPAR $\gamma$ (DEF) and pFR-LUC using the calcium phosphate transfection method. As a reference plasmid for normalization, pcDNA3.1 vector was used. The cells were treated with the indicated concentration of compounds $24 \mathrm{~h}$ after transfection. Following 24 $\mathrm{h}$ of incubation, luciferase assays were performed using a dual-luciferase reporter assay system according to the instructions of the manufacturer (Promega, CA, USA), and the activity was determined in a Victor3 luminometer (PerkinElmer, Inc., MA, USA).

\section{RESULTS}

\section{PPAR $\gamma$ is specifically activated by SP1818}

SP1818, a $\beta$-carboxyethyl-rhodanine derivative, was identified by an improved virtual screening scheme combining ligand-centric and receptor-centric methods using a library of commercially available compounds in SDF format as a PPAR $\gamma$ binder (Choi et al., 2009) and structure is shown in Fig. 1. Rhodanine derivatives showing PPAR stimulating activity were reported previously (Liu et al., 2007), but their structures are different with SP1818. To determine whether SP1818 is capable of activating PPAR $\gamma$, the specific activity of SP1818 as a PPAR $\gamma$ agonist was evaluated in a transient transfection assay in 293T cells. The cells were co-transfected with pFA-Gal4-PPAR $\gamma$ LBD together with pFR-Luc and then treated with SP1818 
and rosiglitazone. SP1818-induced activity increased in a concentration-dependent manner, and the activity of SP1818 at $10 \mu \mathrm{M}$ was similar to that of $1 \mu \mathrm{M}$ rosiglitazone,<smiles>Cc1ccc(COc2ccccc2/C=C2\SC(=S)N(CCC(=O)[O-])C2=O)cc1</smiles>

\section{SP1818}

Fig. 1. Chemical structure of SP1818. SP1818 has a novel scaffold, ( $\beta$-carboxyethyl)-rhodanine. indicating that SP1818 is a partial PPAR $\gamma$ agonist (Fig. 2A). To determine whether SP1818 specifically increases PPAR $\gamma$ activity without affecting PPAR $\alpha$ and PPAR $\delta$ activities, cells were co-transfected pFA-Gal4-PPAR-LBDs with pFR-Luc. The transactivation activity of PPAR $\gamma$ was induced by SP1818 up to $70 \%$ that of rosiglitazone, whereas SP1818 was unable to activate PPAR $\alpha$ or PPAR $\delta$, indicating that SP1818 is a selective PPAR $\gamma$ agonist (Fig. 2B). Since rosiglitazone is able to stimulate glucose uptake in 3T3-L1 adipocytes, we next examined whether SP1818 induce glucose uptake. SP1818 significantly increased 2-deoxyglucose uptake (Fig. 2C).

\section{Adipogenesis is reduced by SP1818}

Several PPAR $\gamma$ agonists induce adipogenesis of preadipocytes, and this is one of the adverse effects of PPAR $\gamma$ agonists. We therefore examined whether SP1818 has an adipogenic effect using 3T3-L1 preadipocyte cells. The cells were treated with the differentiation cocktail, insulin,
(A)

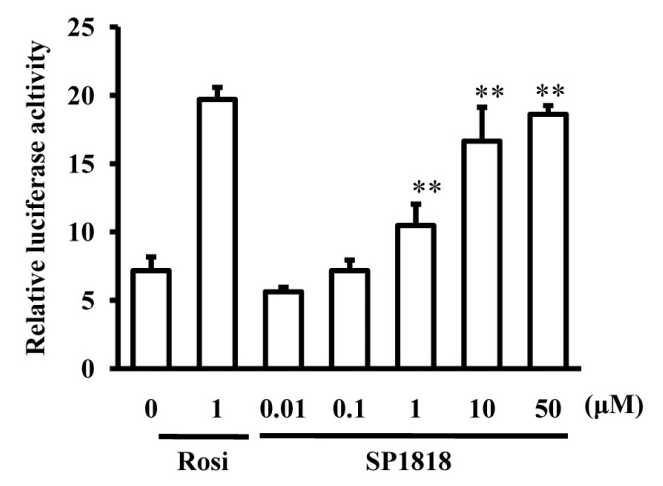

(C)

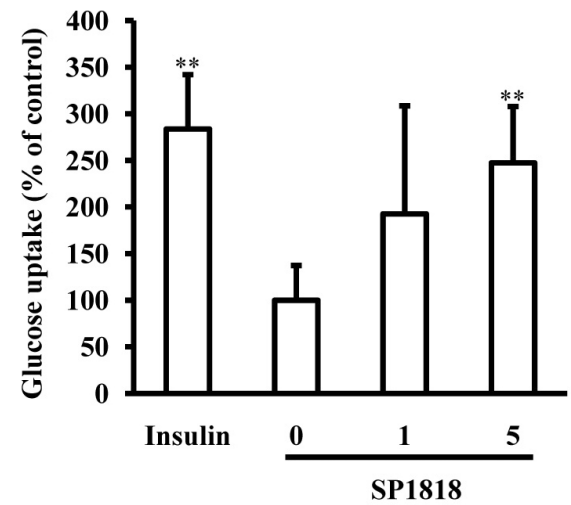

(B)

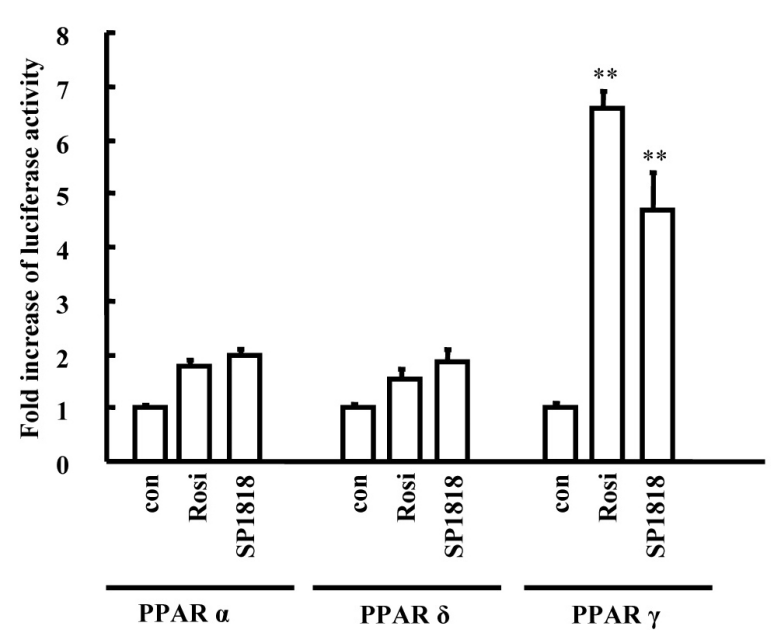

Fig. 2. PPAR $\gamma$ is specifically activated by SP1818. (A) HEK 293T cells were transiently transfected with pFA-Gal4-PPAR $\gamma$-LBD together with pFR-Luc and treated with the indicated concentration of SP1818 or rosiglitazone, respectively. (B) HEK 293T cells were transiently transfected with pFA-Gal4-PPAR-LBDs with pFR-Luc and treated with $10 \mu \mathrm{M}$ SP1818 or $1 \mu \mathrm{M}$ rosiglitazone. Luciferase activity was determined after cell lysis and expressed as fold activation relative to untreated cells. (C) The preadipocyte cell line 3T3-L1 was differentiated with differentiation cocktail and then incubated with the indicated concentration of SP1818 for 30 min. Deoxyglucose uptake was measured using scintillation counter. Values are means \pm S.E.M. of three different experiments. ${ }^{* *} p<0.01$ compared to vector control. 
(A)

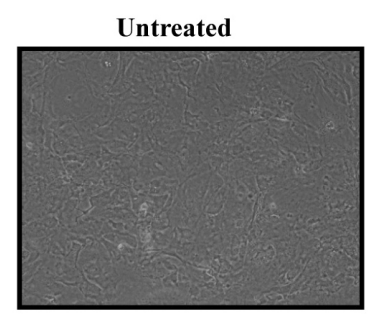

Insulin

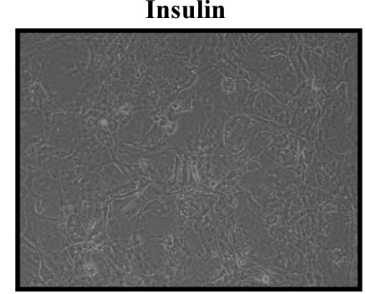

Differentiation cocktail

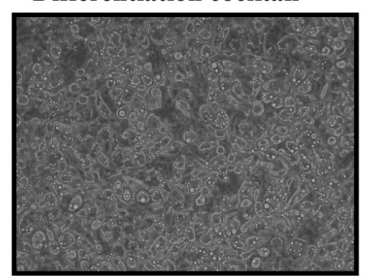

Rosiglitazone

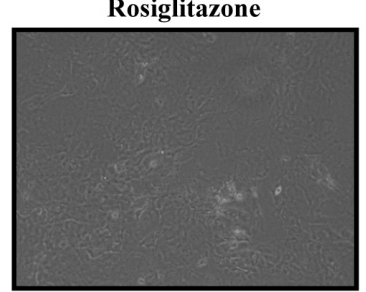

(B)

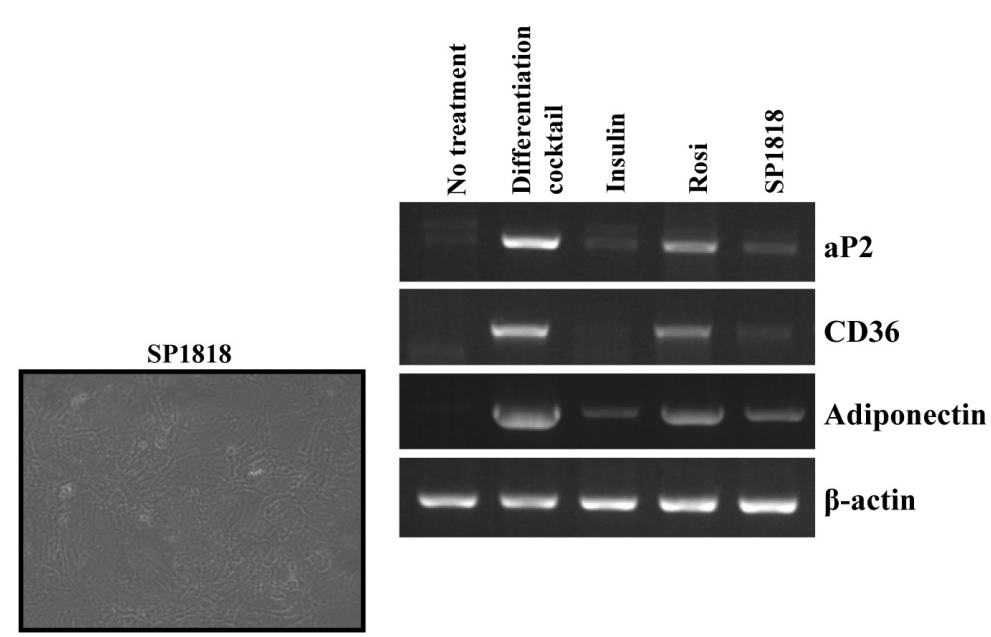

Fig. 3. SP1818 is less adipogenic than rosiglitazone. The preadipocyte cell line 3T3-L1 was differentiated with differentiation cocktail, 1 $\mu \mathrm{M}$ rosiglitazone, $10 \mu \mathrm{M}$ SP1818, or $170 \mathrm{nM}$ insulin. (A) On day seven of differentiation, cells were photographed. (B) The levels of aP2, CD36, and adiponectin mRNA were measured by RT-PCR and the level of beta-actin mRNA was also measured as a loading control.

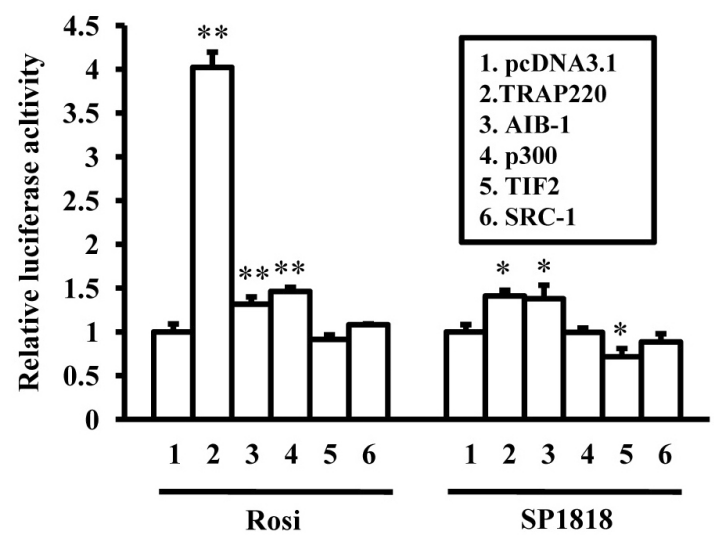

Fig. 4. AIB-1 and TRAP220 are mainly recruited to PPAR $\gamma$ by SP1818. HEK 293T cells were transiently cotransfected with expression vectors for pVP-PPAR $\gamma$ and pFR-Luc reporter construct together with one of the PPAR $\gamma$ cofactor expressing plasmids, including TRAP220, AIB-1, p300, TIF2, and SRC-1. Cells were grown for $24 \mathrm{~h}$ in the presence or absence of SP1818 $(10 \mu \mathrm{M})$ or rosiglitazone $(1 \mu \mathrm{M})$. The recruitment of the cofactor is expressed as fold induction relative to luciferase activity. Values are means \pm S.E.M. of three different experiments in triplicate. ${ }^{*} p<0.05,{ }^{* *} p<0.01$ compared to vector control.

rosiglitazone, or SP1818 for two days, and then culture medium was changed every other day. On day seven of differentiation, lipid droplet levels were photographed and total RNA was isolated. Treatment with SP1818 resulted in less differentiation compared to rosiglitazone treatment
(Fig. 3A). To further confirm this, the expression level of various adipocyte differentiation marker genes was examined, including aP2, CD36, and adiponectin. SP1818 treatment resulted in lower expression levels of all genes tested compared with rosiglitazone treatment (Fig. 3B), indicating that SP1818 has lower adipogenic potential than rosiglitazone.

\section{PPAR $\gamma$ cofactor specificity of SP1818 is different from that of rosiglitazone}

SP1818 showed different adipogenic effects from those of rosiglitazone. Thus, to know the underlying mechanism, we performed a mammalian two-hybrid assay using TRAP220, AIB-1, p300, TIF2, and SRC-1 cofactors fused in-frame to Gal4 binding protein. Cells were co-transfected with one of the cofactor expressing plasmids together with pVP-PPAR $\gamma$ and pFR-luc as a reporter plasmid. Rosiglitazone strongly stimulated interaction of TRAP220 with PPAR $\gamma$, and SP1818 marginally recruited AIB-1 and TRAP220 into PPAR $\gamma$ (Fig. 4), suggesting that SP1818 recruits different cofactors than does rosiglitazone.

\section{DISCUSSION}

Thiazolidinediones (TZDs) are a class of oral hypoglycemic agents to improve insulin sensitivity and the metabolic processes responsible for the development of insulin resistance (Mudaliar and Henry, 2001; Bhatia and Viswanathan, 2006). TZDs lower hyperglycemia by increasing 
peripheral glucose utilization and decreasing hepatic glucose production in both human and in experimental models of insulin resistance (Olefsky, 2000). These effects of TZDs are mediated through PPAR $\gamma$, which is highly expressed in adipose tissue and to a lesser extent in skeletal muscle and liver (Auwerx, 1999). Because the storage of excess energy in adipocytes favors insulin sensitivity, it is not surprise that PPAR has adipogenic activity. However, side effects including weight gain, liver toxicity, fluid retention, and edema need to be surmounted before TZDs are used more widely in the market (Chao et al., 2000; Scheen, 2001; Watkins et al., 2002; Tolman and Chandramouli, 2003). In an effort to find a compound with fewer side effects, we screened candidate compounds. One of the functions of PPAR $\gamma$ is enhancing adipocyte differentiation. Thus we tested whether SP1818 induces the differentiation of adipocytes. The adipogenic activity of SP1818 is less than that of rosiglitazone, which is on the market.

It has been known that cofactors interacts with nuclear receptors and they can either repress or enhance their transcriptional activities (Glass et al., 1997). Both SRC-1 and p300 activators are not specific to PPAR $\gamma$ and the interaction between PGC-1 and PPAR $\gamma$ is ligand-independent both in vitro and in vivo. Although several cofactors interact with PPAR $\gamma$, none of these cofactors seems to be specific for PPAR $\gamma$. Thus, understanding PPAR $\gamma$ liganddependent cofactor profiles is important to estimate the action mechanism of each PPAR $\gamma$ ligand. To elucidate the mechanism underlying SP1818's different profile, we therefore analyzed which of PPAR $\gamma$ 's cofactors (SRC-1, TIF2, AIB-1, p300, and TRAP220) is recruited because direct interactions between PPAR $\gamma$ and SRC-1, TIF2, AIB-1, p300, and TRAP220 have been observed when prostaglandin derivative 15-deoxy-12,14 prostaglandin J2 (15d-PGJ2) is bound to PPAR $\gamma$. Interestingly, when rosiglitazone was bound to PPAR $\gamma$, TRAP220 subunit, a subunit of the thyroid hormone receptor-associated protein (TRAP) transcription coactivator complex, was specifically recruited. However, SP1818 did not interact strongly with TRAP220 subunit compared to rosiglitazone, which may be why SP1818 is less adipogenic than rosiglitazone. PPAR $\gamma$ has an unusually large ligand-binding pocket, which allows it to interact with diverse endogenous and chemical ligands including various fatty acids and the thiazolidinediones ( $\mathrm{Xu}$ and $\mathrm{Li}, 2008$ ). It is therefore possible for SP1818 to recruit an unknown coactivator or a lower amount of coactivators such as AIB-1 and TRAP220, which could explain why SP1818 activates PPAR $\gamma$ only half as much as rosiglitazone. In summary, SP1818 has the ability to stimulate PPAR $\gamma$ activity and is less adipo- genic than rosiglitazone. Since weight gain is one of the side effects of PPAR $\gamma$ agonists, being less adipogenic could make SP1818 a promising candidate drug for the treatment of type II diabetes.

\section{ACKNOWLEDGMENTS}

This research was supported by Sookmyung Women's University Research Grants 2007.

\section{REFERENCES}

Auwerx, J. (1999). PPARgamma, the ultimate thrifty gene. Diabetologia 42, 1033-1049.

Berger, J. P., Akiyama, T. E. and Meinke, P. T. (2005). PPARs: therapeutic targets for metabolic disease. Trends Pharmacol. Sci. 26, 244-251.

Bhatia, V. and Viswanathan, P. (2006). Insulin resistance and PPAR insulin sensitizers. Curr. Opin. Investig. Drugs 7, 891897.

Chao, L., Marcus-Samuels, B., Mason, M. M., Moitra, J., Vinson, C., Arioglu, E., Gavrilova, O. and Reitman, M. L. (2000). Adipose tissue is required for the antidiabetic, but not for the hypolipidemic, effect of thiazolidinediones. J. Clin. Invest. 106, 1221-1228.

Chiarelli, F. and Di Marzio, D. (2008). Peroxisome proliferatoractivated receptor-gamma agonists and diabetes: current evidence and future perspectives. Vasc. Health Risk Manag. 4, 297-304.

Choi, J., Ko, Y., Lee, H. S., Park, Y. S., Yang, Y. and Yoon S. (2009) Identification of ( $\beta$-carboxyethyl)-rhodanine derivatives exhibiting peroxisome proliferator-activated receptor $\gamma$ activity (submitted).

Elsegood, C. L., Chang, M., Jessup, W., Scholz, G. M. and Hamilton, J. A. (2009). Glucose metabolism is required for oxidized LDL-induced macrophage survival: role of PI3K and Bcl-2 family proteins. Arterioscler. Thromb. Vasc. Biol. 29, 1283-1289.

Glass, C. K., Rose, D. W. and Rosenfeld, M. G. (1997). Nuclear receptor coactivators. Curr. Opin. Cell Biol. 9, 222-232.

Hauser, S., Adelmant, G., Sarraf, P., Wright, H. M., Mueller, E. and Spiegelman, B. M. (2000). Degradation of the peroxisome proliferator-activated receptor gamma is linked to ligand-dependent activation. J. Biol. Chem. 275, 1852718533.

Lehmann, J. M., Lenhard, J. M., Oliver, B. B., Ringold, G. M. and Kliewer, S. A. (1997). Peroxisome proliferator-activated receptors alpha and gamma are activated by indomethacin and other non-steroidal anti-inflammatory drugs. J. Biol. Chem. 272, 3406-3410.

Liu, Q., Zhang, Y. Y., Lu, H. L., Li, Q. Y., Zhou, C. H. and Wang, M. W. (2007). Rhodanine derivatives as novel peroxisome proliferator-activated receptor gamma agonists. Acta. Pharmacol. Sin. 28, 2033-2039.

Mudaliar, S. and Henry, R. R. (2001). New oral therapies for type 2 diabetes mellitus: The glitazones or insulin sensitizers. Ann. Rev. Med. 52, 239-257.

Nissen, S. E. and Wolski, K. (2007). Effect of rosiglitazone on 
the risk of myocardial infarction and death from cardiovascular causes. N. Engl. J. Med. 356, 2457-2471.

Olefsky, J. M. (2000). Treatment of insulin resistance with peroxisome proliferator-activated receptor gamma agonists. J. Clin. Invest. 106, 467-472.

Scheen, A. J. (2001). Thiazolidinediones and liver toxicity. Diabetes Metab. 27, 305-313.

Tolman, K. G. and Chandramouli, J. (2003). Hepatotoxicity of the thiazolidinediones. Clin. Liver Dis. 7, 369-379, vi.

Vidal-Puig, A., Jimenez-Linan, M., Lowell, B. B., Hamann, A., Hu, E., Spiegelman, B., Flier, J. S. and Moller, D. E. (1996). Regulation of PPAR gamma gene expression by nutrition and obesity in rodents. J. Clin. Invest. 97, 2553-2561.

Watkins, S. M., Reifsnyder, P. R., Pan, H. J., German, J. B. and Leiter, E. H. (2002). Lipid metabolome-wide effects of the
PPARgamma agonist rosiglitazone. J. Lipid Res. 43, 18091817.

$\mathrm{Xu}, \mathrm{H}$. E. and Li, Y. (2008). Ligand-dependent and -independent regulation of PPAR gamma and orphan nuclear receptors. Sci. Signal 1, pe52.

Zhu, Y., Qi, C., Korenberg, J. R., Chen, X. N., Noya, D., Rao, M. S. and Reddy, J. K. (1995). Structural organization of mouse peroxisome proliferator-activated receptor gamma (mPPAR gamma) gene: alternative promoter use and different splicing yield two mPPAR gamma isoforms. Proc. Nati. Acad. Sci. U S A 92, 7921-7925.

Zoete, V., Grosdidier, A. and Michielin, O. (2007). Peroxisome proliferator-activated receptor structures: ligand specificity, molecular switch and interactions with regulators. Biochimi. Biophys. Acta 1771, 915-925. 